\title{
GERAÇÃO DE MAPAS DE RISCOS NATURAIS EM SANTARÉM - PA: ABORDAGEM BASEADA NA LÓGICA FUZZY
}

\author{
GENERATION OF THE NATURAL RISKS MAPS IN SANTARÉM - PA: AN APPROACH \\ BASED ON FUZZY LOGIC
}

\section{Fábio Ferreira DOURADO ${ }^{1}$, Milena Marília Nogueira de ANDRADE ${ }^{2}$, Cleyton de Carvalho CARNEIRO ${ }^{3}$}

(1) Universidade Federal do Oeste do Pará.E-mail: fabioo.f.dourado@gmail.com (2) Universidade Federal Rural da Amazônia. E-mail: milenamarilia.andrade@gmail.com

(3) Universidade de São Paulo. E-mail: cleytoncarneiro@usp.br

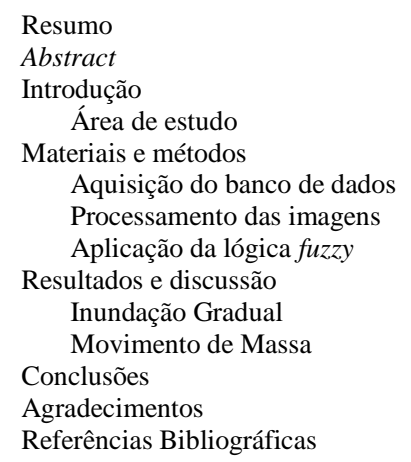

RESUMO - O mapeamento de riscos naturais identifica áreas suscetíveis que apresentam potencial de danos eminentes à população de uma sede urbana. Desse modo, tais mapas representam essencial instrumento para o planejamento urbano, que fomentam a sustentabilidade do ordenamento territorial. Este manuscrito visa gerar mapas de riscos através da análise computacional assistida baseada na técnica fuzzy. A base de dados utilizada reuniu imagens dos sensores ASTER-GDEM e dos radares ALOS-PALSAR e SRTM-InSAR. Estas imagens foram utilizadas para investigar os riscos naturais de inundação gradual e movimento gravitacional de massa na sede urbana de Santarém - PA. Para validação dos resultados obtidos pela fuzzificação foi desenvolvida uma comparação com trabalhos já realizados na área de estudo para os mesmos temas. Para inundação gradual as áreas de riscos alto e moderado são regiões adjacentes as bacias de drenagens na sede urbana e possuem baixa altitude e declividade. Para o evento de movimento de massa as regiões de alto risco são de moderada altitude e alto declive. Os mapas de riscos gerados a partir da lógica fuzzy corroboraram os resultados obtidos com trabalho de campo em estudos anteriores. Tais mapas sugerem que a rotina desenvolvida é eficaz para prever e identificar áreas de risco.

Palavras-chave: lógica fuzzy; riscos naturais; inundação; movimento de massa; Santarém.

\begin{abstract}
The mapping of natural hazards identifies susceptible areas for imminent harm to the population of an urban regions. These maps represent an essential tool for urban planning to promote sustainability of land use. This manuscript aims to generate risk maps using assisted computational analysis based on fuzzy technique. The database was composed by images of ASTER-GDEM sensor and ALOS-PALSAR radar. These images were used to study natural hazards of flooding and gradual gravitational mass movement in the urban region of Santarém city, in Pará state, Brazil. The validation of the results obtained by fuzzification was developed from a comparison with some fieldwork previously developed in the study area for the same subjects. The high and moderate risk areas for gradual flood are adjacent regions to drainage basins and have low altitude and steepness. The results about mass movement event indicated that high-risk areas have moderate altitude and high slope. The risk maps generated from the fuzzy logic corroborate results from previous studies. These maps suggest that the developed routine is effective to predict and identify risk areas.
\end{abstract}

Keywords: Fuzzy Logic; natural risk; flood; mass movement; Santarém.

\section{INTRODUÇÃO}

Risco é a probabilidade de consequências prejudicais e/ou perdas sociais, econômicas ou ambientais resultantes da interação entre riscos naturais e os sistemas humanos (UNDP, 2004). O mapeamento de áreas de risco é necessário sobretudo no cenário de eventos hidrológicos extremos na Amazônia.

Contribuições de mudanças globais no clima e conexão com o fenômeno La Niña estão relacionados com a intensificação das precipitações no oeste da bacia amazônica.
Consequentemente, este incremento hidrológico contribui para o aumento das inundações e da deflagração de movimentos de massa (Tominaga, 2007; Espinoza et al., 2014).

As inundações são a tipologia de desastre naturais mais frequentes no Brasil e em especial na região Amazônica (Silva Júnior \& Szlafsztein, 2010a), representando cerca de $59 \%$ das ocorrências totais entre os desastres, seguido dos movimentos gravitacionais de massa com 14\% dos registros (Silva, 2009). 
No estado do Pará, no período de 1991 a 2012, foram registrados 256 registros oficiais para inundação gradual, com 614.707 pessoas afetadas, 117.027 desalojadas, 38.911 desabrigadas, 30.100 enfermas, 1.798 feridas e 29 mortas (CEPED; UFSC, 2013). Desse total de registros, cerca de $38 \%$ ocorreram na mesorregião do Baixo Amazonas. A maior parte destes eventos foi registrado no ano de 2009, ressaltando o primeiro semestre com os meses de abril e março detentores dos maiores números de ocorrências, justificado pelas altas taxas fluviométricas dos rios da região (BRASIL, 2009; CEPED; UFSC, 2013).

Os movimentos gravitacionais de massa contabilizaram no estado do Pará, no mesmo período das inundações, um total de 117 registros, com 5.131 pessoas afetadas, 230 desalojadas, 215 desabrigadas, 9 feridas e 4 mortas. Esses registros também foram maiores no ano de 2009, com a alta ocorrência no primeiro semestre do ano, com as maiores frequências nos meses de maio e fevereiro, respectivamente (BRASIL, 2009; CEPED; UFSC, 2013).

Os estudos de casos de desastres naturais e de técnicas para geração de mapas de riscos vêm sendo crescentes no Brasil e em especial na região amazônica nas ultimas décadas. Estas técnicas têm utilizado nas elaborações de mapas de risco atributos naturais e antrópicos. No caso das classificações computacionais assistidas destacam-se atributos de: altitude, declividade, reflectância, rede de drenagem, infraestrutura urbana e metodologias qualitativas e participativas (Silva Júnior \& Szlafsztein, 2010a, 2102; Andrade \& Szlafsztein, 2013, 2015; Dourado \& Andrade, 2015).

Segundo Fernandes \& Miola (2013) devido à complexidade no mapeamento de risco para esses eventos, tornam-se necessários estudos com base técnica. Nesse sentido, destaca-se a possibilidade de modelar a geomorfologia das áreas de riscos através da aplicação dos métodos fornecidos pelas geotecnologias, bem como a utilização da lógica fuzzy na geração desses modelos (Ercanoglu \& Gokceoglu, 2004; Jiang et al., 2009).

Tem sido utilizado principalmente Sistema de Informação Geográfica (SIG) e técnicas de sensoriamento remoto em conjunto com modelagens e algoritmos matemáticos em
Modelos Digitais de Elevação (MDE) (Vieira et al., 2005; Hora \& Gomes, 2009; Silva Júnior \& Szlafsztein, 2010a; Bathrellos et al., 2012; Sadeck et al., 2012; Pôssa \& Ventorini, 2014; Prina \& Trentin, 2014; Andrade \& Szlafsztein, 2015; Dourado \& Andrade, 2015).

Trabalhos anteriores ressaltam a importância da utilização de métodos matemáticos para previsão e avaliação de riscos a inundação (Nayak et al., 2005). Contudo a inserção da incerteza na resolução do problema através da lógica fuzzy ainda é incipiente para o mapeamento das inundações (Jiang et al., 2009).

Para mapear o risco a movimento de massa os atributos frequentes são: curvatura vertical, curvatura horizontal, forma de encosta, declividade, geologia, pedologia, geomorfologia, cobertura vegetal, nível pluviométrico, tipo de solo e uso do solo (Saito, 2004; Pfaltzgraff, 2007; Vestana, 2010; Canavesi et al., 2013; Simões \& Oliveira, 2014; Dourado \& Andrade, 2015; Nogueira \& Machado, 2015; Pinto et al., 2015).

Desse modo a lógica fuzzy se tornou uma ferramenta importante quando não se tem dados estatísticos suficientes e há dificuldade de aplicação de modelos matemáticos por falta de dados. A utilização desta técnica em uma base de dados integrada em ambiente de SIG tem sido utilizada frequentemente (Ercanoglu \& Gokceoglu, 2004).

O objetivo desse trabalho é mapear as áreas de risco na área urbana de Santarém à inundação gradual e a movimento gravitacionais de massa utilizando a lógica fuzzy.

\section{Área de Estudo}

A área urbana de Santarém está inserida na mesorregião do Baixo Amazonas no estado do Pará - Brasil, sob as coordenadas geográficas $02^{\circ} 25^{\prime} 30^{\prime \prime}$ S e $54^{\circ} 42^{\prime} 50^{\prime \prime} \mathrm{W}$ (Figura 1).

O município de Santarém é considerado um polo para a região do oeste do Pará em questões econômicas, educacionais, saúde e em infraestrutura (Oliveira et al., 2000; Oliveira, 2008; Andrade \& Szlafsztein, 2015).

A população da área de estudo é de aproximadamente 290.521 habitantes (IBGE, 2014).

Atualmente a malha urbana está subdividida em 48 bairros, segundo o plano diretor do município. 


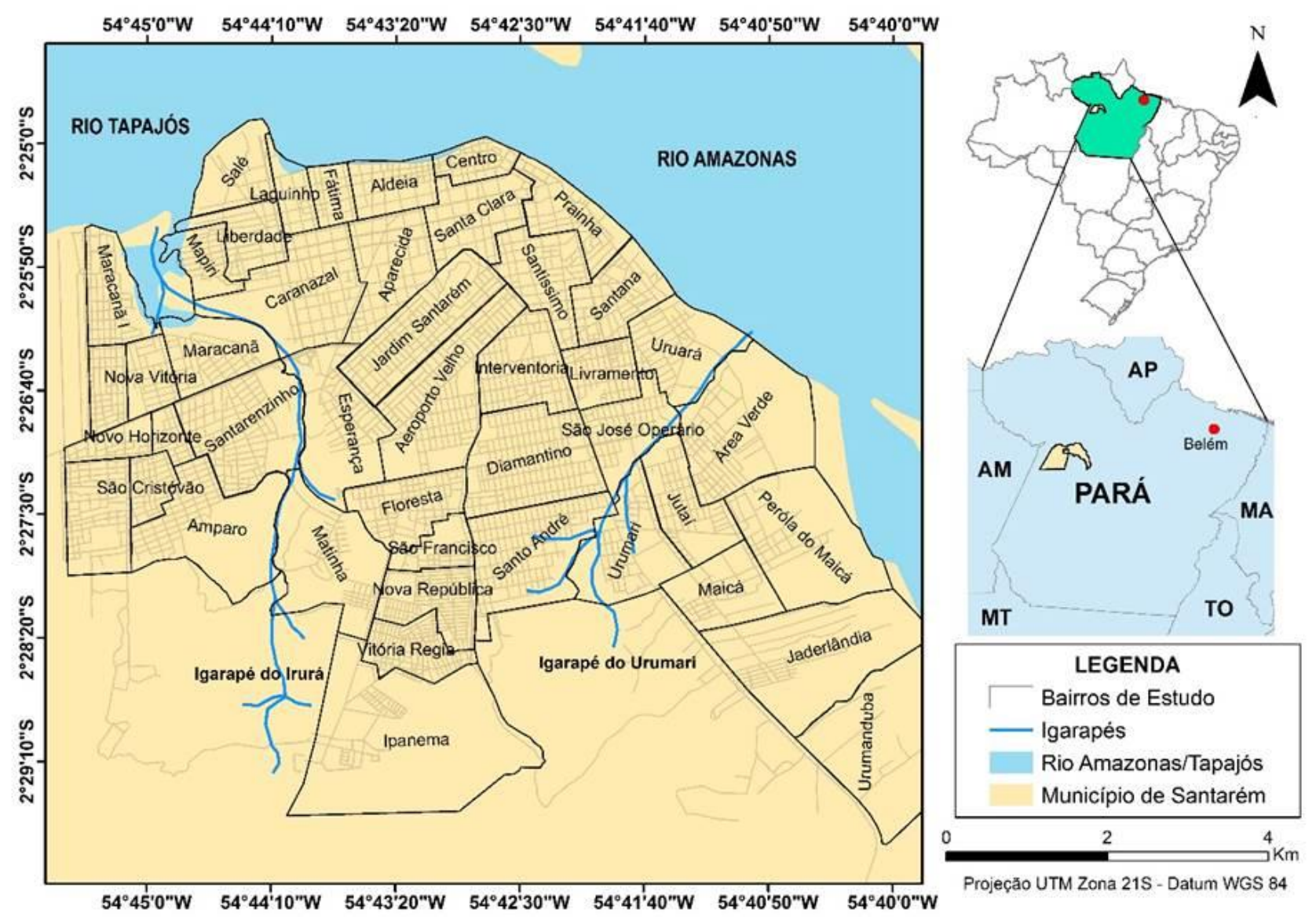

Figura 1 - Área de estudo, sede urbana de Santarém - PA.

Santarém situa-se na confluência dos rios Amazonas e Tapajós, na Bacia do Amazonas. No ano de 2009 foi registrada a maior inundação lenta da história até o momento no município de Santarém, ao qual os níveis dos rios alcançaram a marca de $831 \mathrm{~cm}$ no mês de maio (CPRM, 2012), resultando num total de 78.827 pessoas afetadas e 584 pessoas desabrigadas (BRASIL, 2009).

A segunda e terceira maiores inundações, ocorrentes nos anos de 2014 e 2012, foram de níveis fluviométricos de 813 e $794 \mathrm{~cm}$, respectivamente (ANA, 2014). Os decretos de situação de emergência municipais e/ou estaduais contabilizam seis registros na sede urbana de Santarém para os últimos 21 anos (1991-2012) (CEPED; UFSC, 2013).

Nas áreas mais centrais da cidade, nos morros-testemunhos do relevo dissecado da formação Alter do Chão, as escarpas com alta declividade foram marcadas por movimentos de massa (Andrade \& Szlafsztein, 2013).

Segundo dados da CEPED; UFSC, (2011; 2013), no período de 1991 a 2012, apenas 1 (um) decreto de situação de emergência foi feito no município de Santarém para movimento de massa, sendo este registrado no ano de 2008.

\section{MATERIAIS E MÉTODOS}

Três etapas principais encadearam a sequência metodológica aplicada nesta pesquisa.

\section{Aquisição do Banco de Dados}

Esta etapa foi a de aquisição do banco de dados, disponibilizado gratuitamente: imagem de satélite, imagens de radares e os arquivos vetoriais da área de estudo (malha urbana, limite dos bairros, limite municipal, regional e hidrografia).
A imagem de radar ALOS-PALSAR da cena 227-62 do ano de 2011 e a do satélite ASTERGDEM correspondente à cena SA-21-Z-B de 2009 foram adquiridas na base de dados TOPODATA - Instituto Nacional de Pesquisa Espaciais (INPE).

O MDE obtido a partir do radar interferométrico SRTM-InSAR no ano de 2008 foi adquirido no site do EMBRAPA-RELEVO - Empresa Brasileira de Pesquisa Agropecuária. 
Os arquivos vetoriais da área de estudo foram obtidos no site do IBGE.

\section{Processamento das Imagens}

A segunda etapa envolveu o registro da imagem de radar em relação às imagens ASTER-GDEM. Este procedimento visou melhorar a precisão do posicionamento geográfico, inconsistente nas imagens ALOSPALSAR.

Foram utilizados 60 pontos de controle obtidos com referência nas imagens ASTERGDEM para registrar a imagem ALOSPALSAR.

\section{Aplicação da Lógica Fuzzy}

A terceira etapa foi a aplicação da lógica fuzzy proposta por Zadeh (1965) em ambiente de SIG (Taubenbock et al., 2008).

Para isso foi feito a fuzzyficação dos seguintes atributos: (i) altitude, (ii) declividade $\mathrm{e}$ (iii) rede de drenagem (Andrade \& Szlafsztein, 2015; Silva et al., 2015), a partir do
MDE/SRTM-InSAR; e (iv) refletância, a partir da imagem ALOS-PALSAR.

Tais atributos foram "fuzzyficados" levando em consideração as funções realçam, em cada variável, os valores relacionados aos riscos naturais.

O próximo passo foi definir a função de pertinência $(\mu)$, a qual expressa a transição gradual do elemento do conjunto universo $\mathrm{X}$ de pertencer ou não ao intervalo dos números reais de 0 a 1.

(Equação 1).

$$
A=\{[x, \mu \mathrm{A}(x)] \mid x \in \mathrm{X}\} \text {, onde } \mu \mathrm{A}: \mathrm{X} \rightarrow[0,1](1)
$$

Posteriormente foram definidos os spreads e os midpoints e, em seguida, definido as variáveis (Figuras 2 e 3) aplicadas para cada mapa de risco natural, conforme a Tabela 1 , e então gerada a função de pertinência de cada uma delas.
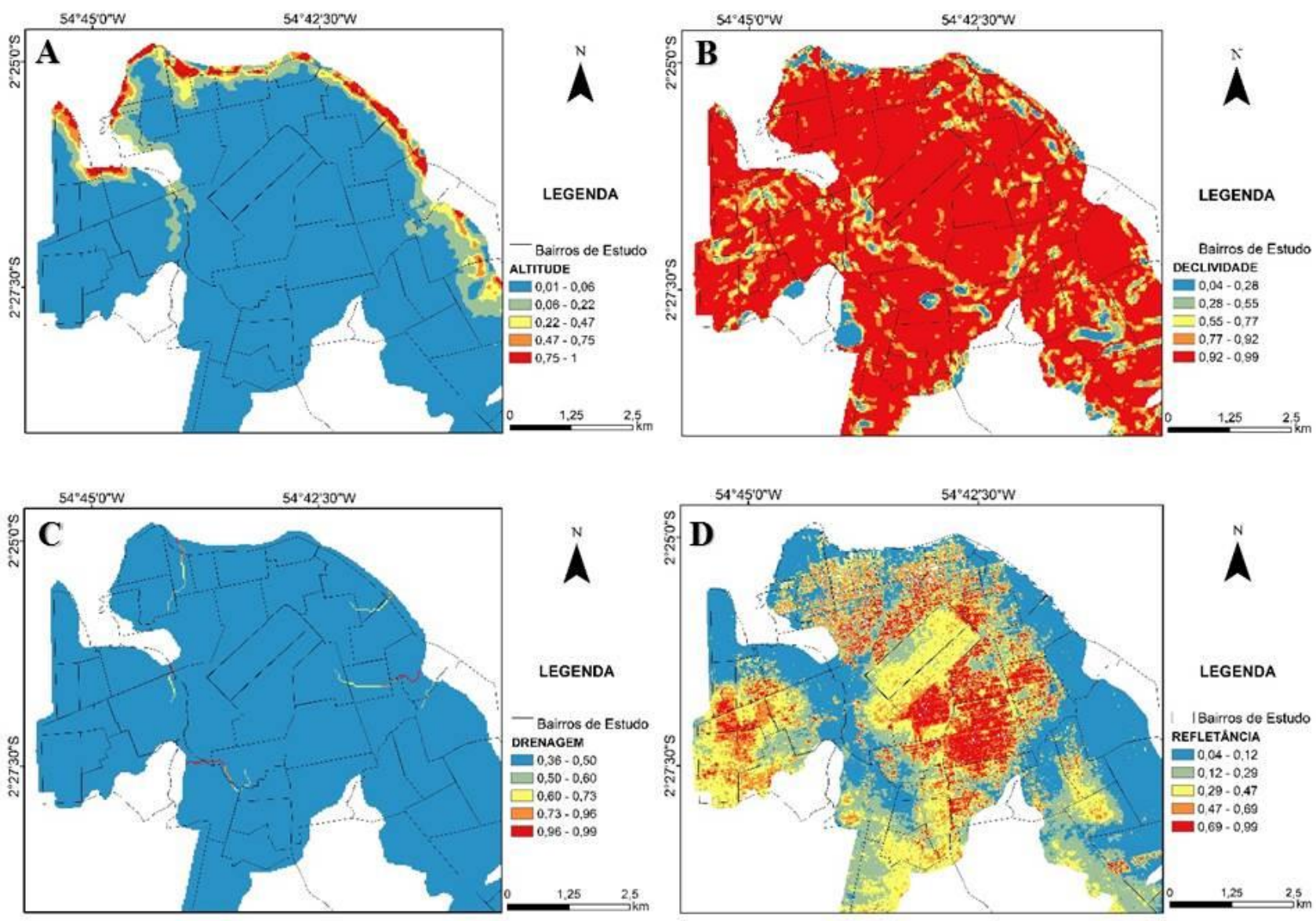

Figura 2 - Variáveis para o mapa de inundação gradual:A) mapa com baixos valores de altitude (vermelho); B) mapa com baixos valores de declividade (vermelho); C) mapa com alta concentração de rede de drenagem (laranja/azul); D) mapa de alta refletância de radar para destaque de áreas ocupadas (vermelho). 

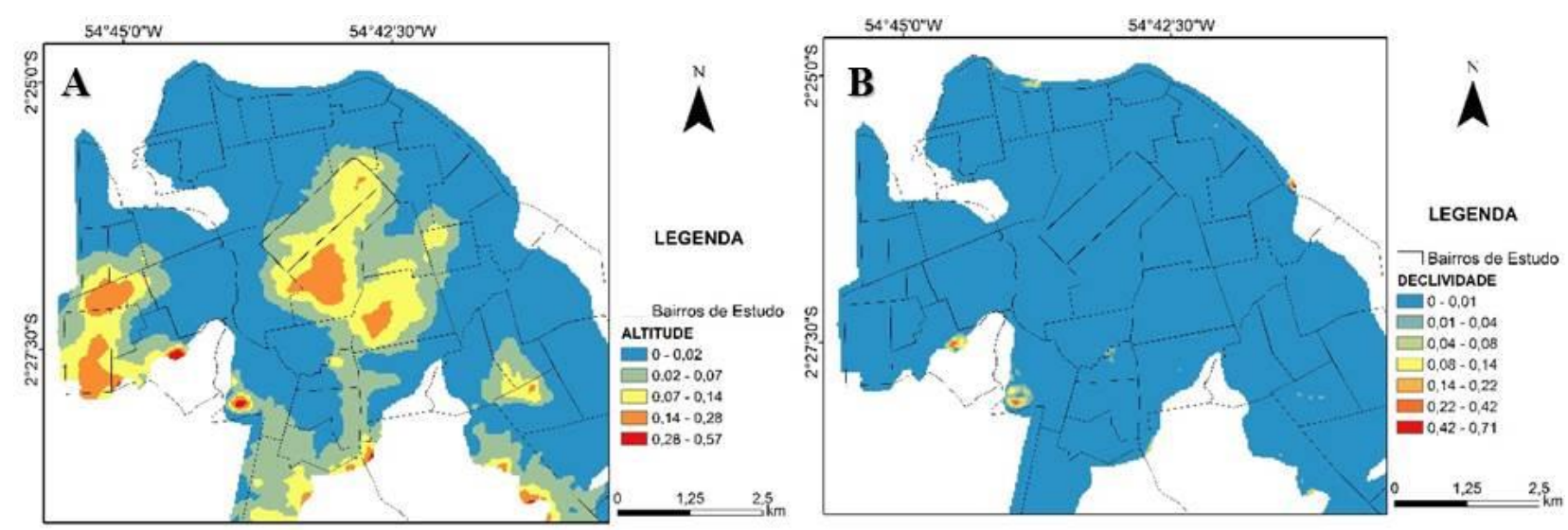

Figura 3 - Variáveis para o mapa movimento de massa: A) mapa com altos valores de altitude (laranja e vermelho); B) mapa com altos valores de declividade (laranja e vermelho).

Tabela 1 - Atributos e valores utilizados para a geração dos mapas de riscos.

\begin{tabular}{|c|c|c|c|c|c|c|}
\hline $\begin{array}{c}\text { RISCO } \\
\text { NATURAL }\end{array}$ & ATRIBUTOS & IMAGENS & $\begin{array}{c}\text { FUNÇÃO DE } \\
\text { PETRINÊNCIA } \\
\text { FUZZY } \\
\end{array}$ & SPREADS & MIDPOINTS & $\begin{array}{c}\text { FUNÇÃO DE } \\
\text { INTEGRAÇÃO } \\
\text { FUZZY } \\
\end{array}$ \\
\hline \multirow{4}{*}{$\begin{array}{l}\text { INUNDAÇÃO } \\
\text { GRADUAL }\end{array}$} & Altitude & \multirow{3}{*}{$\begin{array}{l}\text { MDE SRTM } \\
\text { InSAR }\end{array}$} & \multirow{2}{*}{ Small } & & \multirow{2}{*}{10} & \multirow{4}{*}{ FUZZY AND } \\
\hline & Declividade & & & & & \\
\hline & Drenagem & & \multirow{5}{*}{ Large } & & 4300 & \\
\hline & Refletância & ALOS-PALSAR & & 5 & 6134 & \\
\hline \multirow{3}{*}{$\begin{array}{l}\text { MOVIMENTO } \\
\text { DE MASSA }\end{array}$} & Altitude & \multirow{2}{*}{$\begin{array}{l}\text { MDE SRTM } \\
\text { InSAR }\end{array}$} & & & 40 & \multirow{3}{*}{ FUZZY GAMMA } \\
\hline & Declividade & & & & 93 & \\
\hline & Refletância & ALOS-PALSAR & & & 6134 & \\
\hline
\end{tabular}

Por fim os mapas finais foram gerados em escala de 1:45.000 a partir integração das variáveis fuzzyficadas por meio de duas funções de integração fuzzy: Fuzzy And e Fuzzy Gamma.

A função Fuzzy And (FA) é definida a partir de um conjunto clássico de valores $(1,0)$. A intercessão significa uma sequência de "e", e é obtida através da utilização do operador MIN (Equação 2).

$$
\mu=\operatorname{MIN}\left(\mu_{\mathrm{A}}, \mu_{\mathrm{B}}, \mu_{\mathrm{C}} \ldots\right)
$$

onde $\mu A$ é o valor de pertinência para o mapa $\mathrm{A}$ em determinada localização, o $\mu B$ é o valor para o mapa B.

A função Fuzzy Gamma (FG) é definida a partir das funções "Fuzzy-Produto" e "FuzzySoma" de acordo com a equação abaixo (Bonham-Carter, 1994) (Equação 3).

$$
F G=(\text { Fuzzy }- \text { Soma })^{\gamma} \cdot(\text { Fuzzy }- \text { Produto })^{1-\gamma}
$$

onde $\gamma=$ parâmetro que varia de 0 a 1 .

\section{RESULTADOS E DISCUSSÃO}

\section{Inundação Gradual}

O mapa gerado para o evento de inundação gradual apontou ao todo 14 bairros com risco de escala moderada e alta (Figura 4). Todos esses bairros estão situados às margens dos rios em regiões com baixas altitudes e suaves declividades apresentando-se nas regiões norte e noroeste na área de estudo (Andrade \& Szlafsztein, 2013).
Os bairros apontados com níveis de risco baixos perfazem a maior parte na área de estudo.

Estes bairros situam-se em regiões topograficamente mais elevadas. São áreas da cidade que não foram atingidas em inundações excepcionais já registradas anteriormente. Estes bairros se localizam na parte do centro da sede urbana. 


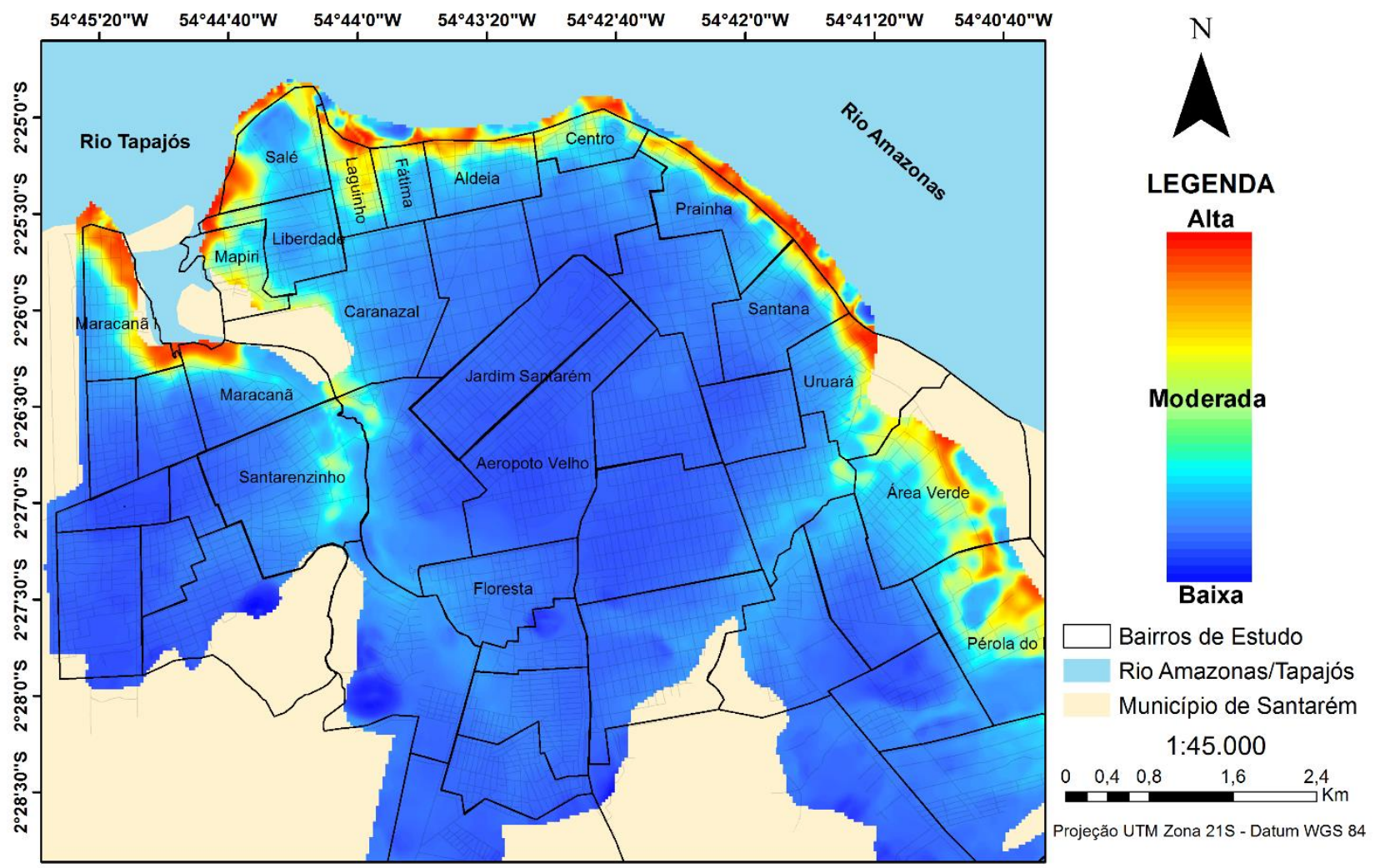

Figura 4 - Mapa de risco a inundação gradual apontando 14 bairros com risco de nível moderado e alto.

O mapa de risco aponta 14 bairros em situação de risco moderado a alto. Destes, 8 foram confirmados pelo mapa participativo proposto por Andrade \& Szlafsztein (2013; 2015), com mesmos níveis de riscos, são eles: Aldeia, Centro, Maracanã, Maracanã I, Uruará, Área Verde, Pérola do Maicá e Mapiri. Estes ocupam uma área de aproximadamente 250 ha de risco alto e de 240 ha de risco moderado (Andrade \& Szlafsztein, 2013). Para o mapa de risco, os bairros com nível de risco alto representam uma área de aproximadamente 90 ha e de 70 ha para risco moderado.

O bairro do Caranazal, descrito pelo mapa participativo, foi classificado com nível moderado a alto, já pelo mapa de risco foi classificado como baixo. Outros 6 bairros apresentaram pelo mapa de risco nível moderado e alto, são eles: Salé, Laguinho, Fátima, Liberdade, Prainha e Santana, com cerca de 25 ha para nível moderado e 30 ha para alto.

A região afetada pela inundação gradual corresponde cerca de $37 \%$ da área total da área urbana, afetando aproximadamente 97 mil habitantes, e grande parte dessa região compreende ao polo de comércio do município (IBGE, 2011).

Publicações recentes feitas por Dourado \&
Andrade $(2014 ; 2015)$ apontam que o período de inundação gradual na região se iniciam no mês de abril e estendem-se até meados de julho, sendo nos meses de maio e junho registrado os maiores níveis dos rios nas grandes inundações ocorridas (ANA, 2014).

As populações habitantes dessas regiões afetadas relatam diversas perdas no período de inundação, dentre elas: perdas materiais (móveis, eletrodomésticos e casa de famílias), perdas econômicas (diminuição do lucro do comércio, por parte do acesso limitado ao local e também perdas agrícolas), o surgimento de enfermidades relativas ao consumo da água e a aparição de animais peçonhentos no local (Andrade \& Szlafsztein, 2015).

\section{Movimento de Massa}

O mapa gerado para o evento de movimento gravitacional de massa apontou 11 bairros com risco moderado e alto (Figura 5).

Estes estão situados nas interfaces de relevo de colina, em regiões com valores médio a altos de altimetria e declividade, estando estes distribuídos por quase toda sede urbana, se concentrados no centro, região oeste, sul e sudeste.

A maioria dos bairros apresentaram nível de risco baixo - por estes se situarem em regiões com menores altitude e declividade. 


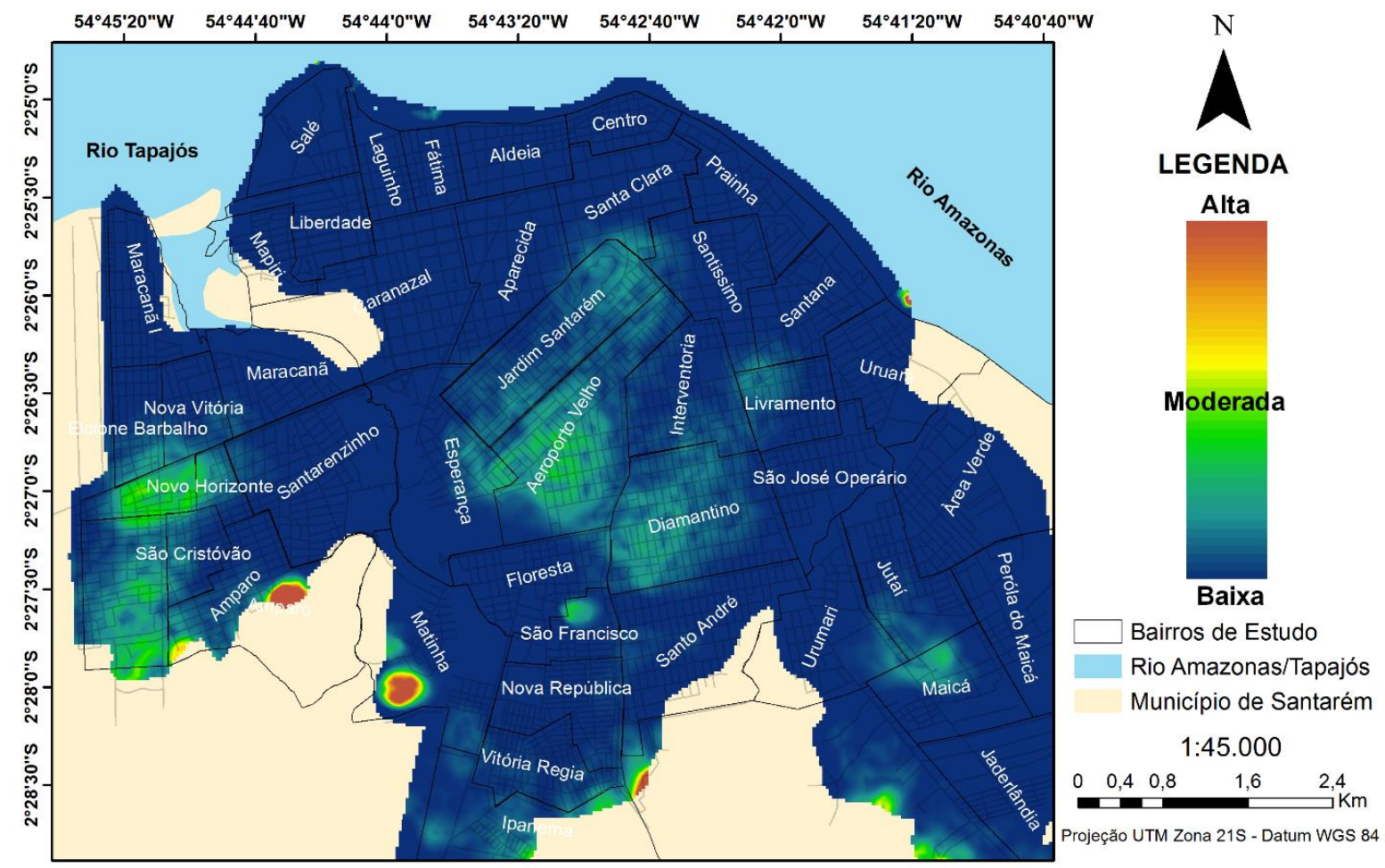

Figura 5 - Mapa de risco a movimento de massa apontando 11 bairros com risco de nível moderado e alto.

Um trabalho publicado recentemente por Nogueira \& Machado (2015), apresenta uma carta de susceptibilidade a movimento de massa no município de Santarém, sob escala de 1:1.000.000, apontando a sede urbana de estudo como de baixa amplitude e sendo este classificada como de nível baixo para tal evento.

Contudo em escala local 11 bairros foram declarados pelo mapa de risco com nível moderado e alto, 2 deles apontaram nível alto para as regiões de morro, localizados nos bairros Amparo e Matinha.

Os autores Dourado \& Andrade (2015), descreveram no bairro Amparo o morro do Índio, localmente conhecido como "serra do Índio", com presença de movimento de massa dos tipos: queda de bloco, corrida de massa e escorregamento, isso devido a presença de escarpas com declividade moderada a íngremes. E ainda, por ser constituídos basicamente de rochas sedimentares de fácil desintegração pertencentes à Formação Alter do Chão, tais como: arenito avermelhados, siltitos, conglomerados e brechas (Daemon, 1975).

No bairro da Matinha está situado o "morro da Matinha", sendo este também apontado como de risco alto pelo mapa de risco. Os 9 bairros que apresentaram risco de nível moderado são: Aeroporto Velho, Maicá, Jutai, Uruará, Diamantino, São Francisco, Novo Horizonte, Elcione Barbalho e Matinha, estes estão em regiões com médias altitudes e declividades.

\section{CONCLUSÃO}

A geração e interpretação de mapa de risco aos eventos de inundação gradual e ao movimento gravitacional de massa a partir da lógica fuzzy é apontada como ferramenta eficiente para previsão e classificação das áreas que apresentam riscos a esse evento, tal ferramenta pode ser utilizada no complemento de outras técnicas de mapeamento.

A disponibilidade de dados gratuitos em plataformas de livre acesso possibilitam, assim, ferramenta acessível e de baixo custo, que podem ser adquiridos por prefeituras e entidades que atuam em zonas de riscos naturais na Amazônia.

A lógica fuzzy pode ser usada durante a fase exploratória, para o mapeamento de possíveis áreas de risco.

Posteriormente, devendo ser feito o trabalho in loco para a devida validação das áreas indicadas como maior risco e para a aquisição de informações adicionais ou desenvolvimento de retificações. 


\section{AGRADECIMENTOS}

O primeiro autor agradece a Pró-Reitoria de Ensino de Graduação - PROEN da Universidade Federal do Oeste do Pará - UFOPA, pela concessão da bolsa de mobilidade acadêmica externa temporária nacional, edital no 003/2014-PROEN/UFOPA de 11 de março de 2014.

Gratidão ao Prof. Dr. Cleyton de Carvalho Carneiro pelos ensinamentos valiosos sobre classificação supervisionada utilizando Lógica Fuzzy, e também a Universidade de São Paulo USP pela recepção e suporte para desenvolvimento destes resultados.

\section{REFERÊNCIAS}

ANA - AGÊNCIA NACIONAL DAS ÁGUAS. Dados fluviométricos da estação (17900000) de Santarém - PA. Disponível em: http://mapashidro.ana.gov.br/Usuario/ Dado Pesquisar.aspx ?est=22454440. Acessado em: 10Ago2014. 2014.

ANDRADE, M.M. \& SZLAFSZTEIN, C.F. Mapa de Inundação como Instrumento de Planejamento Urbano Estudo de caso na sede urbana de Santarém. SIMPÓSIO DE GEOLOGIA DA AMAZÔNIA $\mathbf{1 3}^{\circ}$. 2013. Belém. Resumos...Belém: Sociedade Brasileira de Geologia, 2013.

ANDRADE, M.M. \& SZLAFSZTEIN, C.F. Community participation in flood mappingin in the Amazon through interdisciplinary methods. Natural Hazards. ISSN: 0921030X. doi:10.1007/s11069-015-1782-y. 2015.

BATHRELLOS, G.D.; PAPANASTASSIOU, K.; SKILODIMOU, H.D.; PAPANASTASSIOU, D.; HOUSIANITIS, K.G. Potential suitability for urban planning and industry development using natural hazard maps and geological-geomorphological parameters. Environ Earth Sci, v. 66, p. 537-548. 2012.

BONHAM-CARTER, G.F. Geographic information systems for geoscientists, modeling with GIS. Elsevier. Ontario Pergamon, v. 13, p. 398. 1994.

BRASIL. Banco de dados de registros de desastres: sistema integrado de informações sobre desastre - S2ID. Disponível em: http://s2id.integracao.gov.br. Acesso em: 20Fev2016. 2009.

CANAVESI, V.; CAMARINHA, P.I.M.; ALGARVE, V.R.; CARNEIRO, R.L.C. DE.; ALVALÁ, R.C.S. DOS. Análise da susceptibilidade a deslizamentos de terra: estudo de caso de Paraibuna, SP. In: SIMPÓSIO BRASILEIRO DE SENSORIAMENTO REMOTO XVI. Resumos Expandidos... Foz do Iguaçu, PR. INPE: Instituto Nacional de Pesquisas Espaciais, 2013, p. 5252-5258.

CEPED; UFSC. Atlas brasileiro de desastres naturais. 19912010. Volume Pará. Florianópolis: UFSC. Disponível em: http://150.162.127.14:8080/atlas/Atlas\%20Para\%202.pdf. Acessado em: 15Ago2013. 2011.

CEPED; UFSC. Atlas brasileiro de desastres naturais. 19912012. Volume Pará. Florianópolis: UFSC. Disponível em: http://150.162.127.14:8080/atlas/Atlas\%20Para.pdf.

Acessado em: 15Ago2013. 2013.

CPRM - COMPANIA DE PESQUISA DE RECURSOS MINERAIS - SERVIÇO GEOLÓGICO DO BRASIL. Monitoramento Hidrológico de 2012 - Boletim Abril. Manaus: SIPAM. 2012.

DAEMON, R.F. Contribuição à datação da Formação Alter do Chão, bacia do Amazonas. Revista Brasileira de Geologia, v. 5 , p. $58-84.1975$.

DOURADO, F.F. \& ANDRADE, M.M. Comparação entre o mapa de ameaça à inundação lenta em Santarém (PA) e o conteúdo jornalístico virtual publicado. In: $47^{\mathrm{a}}$ CONGRESSO BRASILEIRO DE GEOLOGIA. 2014. Salvador. Resumos... Salvador: Sociedade Brasileira de Geologia, 2014.

DOURADO, F.F. \& ANDRADE, M.M. Mapeamento dos perigos naturais na sede urbana de Santarém - PA. 2015. Bento Gonçalves In: CONGRESSO BRASILEIRO DE
GEOLOGIA E ENGENHARIA $15^{\circ}$. Resumos Expandidos... Bento Gonçalves, RS: Associação Brasileira de Geologia e Engenharia e Ambiental, 2015.

ERCANOGLU, M. \& GOKCEOGLU, C. Use of fuzzy relations to produce landslide susceptibility map of a landslide prone area (West Black Sea Region, Turkey). Elsevier. doi:10.1016/j.enggeo.2004.06.001. v. 75, p. 229250. 2004.

ESPINOZA, J.C.; MARENGO, J.A.; RONCHAIL, J.M.C.; FLORES, L.N.; GUYOT, J.L. The extreme 2014 flood in south-western Amazon basin: the role of tropical-subtropical South Atlantic SST gradient. Envionmental Resarch Lettes, v. 9, p. 1-9. 2014.

FERNANDES, N.S. DA. \& MIOLA, C.A. Geoprocessamento aplicado no mapeamento de áreas com risco de inundação em Santa Maria-RS. In: SIMPÓSIO BRASILEIRO DE SENSORIAMENTO REMOTO XVI. 2013, Foz do Iguaçu Resumo Expandido... Foz do Iguaçu: Instituto Nacional de Pesquisas Espaciais, 2013, p. 4920-4927.

HORA, S.B. \& GOMES, R.L. Mapeamento e avaliação do risco a inundação do rio cachoeira em trecho da área urbana do município de Itabuna/BA. Sociedade e natureza, Uberlândia, v. 21, p. 57-75. 2009.

IBGE - INSTITUTO BRASILEIRO DE GEOGRAFIA E ESTATÍSTICA. Sinopse do Censo Demográfico. Rio de Janeiro. Disponível em: http://www.ibge.gov.br/home/ estatística/população/censo2010/default_sinopse.shtm. Acessado em: 11Jun2014. 2011.

IBGE - INSTITUTO BRASILEIRO DE GEOGRAFIA E ESTATÍSTICA. Dados estatístisticos municipais de Santarém - PA. Disponível em: http://cidades.ibge. gov.br/xtras/uf.php?lang=\&coduf=15\&search=pa. Acesso em: 20Mai2014. 2014.

JIANG, W.; DENG, L.; CHEN, L.; WU, J.; LI, J. Risk assessment and validation of flood disaster based on fuzzy mathematics. Elsevier, v. 19, p. 1419-1245. 2009.

NAYAK, P.C.; SUDHEER, K.P.; RAMASASTRI, K.S. Fuzzy computing based rainfall - runoff model for real time flood forecasting. Hydrological Processes, v. 19, p. 955-968, 2005.

NOGUEIRA, A.C. \& MACHADO, M.F. Carta de suscetibilidade a movimentos gravitacionais de massa e inundação do município de Santarém - Pará. In: CONGRESSO BRASILEIRO DE GEOLOGIA DE ENGENHARIA E AMBIENTAL $15^{\circ}$. 2015, Bento Gonçalves. Resumos Expandidos... Bento Gonçalves: Associação Brasileira de Geologia de Engenharia e Ambeintal, 2015.

OLIVEIRA, J.M. Expansão Urbana e Periferização de Santarém-PA, Brasil: Questões para o Planejamento Urbano. In: COLÓQUIO INTERNACIONAL DE GEOCRÍTICA X, 2008. Barcelona. Resumos Expandidos... Espanha: Univesitat de Barcelona, 2008.

OLIVEIRA, J.R.; PASTANA, J.M.; NAVEGANTE, P.S.; SCHALKEN, C.G. Caracterização Hidrogeológica da Cidade de Santarém e das Vilas de Mojuí dos Campos e Alter-DoChão com Proposta Técnica para a Perfuração de Poços Tubulares Profundos. In: JOINT WORLD CONGRESS ON 
GROUNDWATER, $1^{\text {st }}, \quad 2000$, Belém. Resumos Expandidos... Pará: Associação Brasileira de Águas Subterrâneas, 2000.

PFALTZGRAFF, P.A.S. DOS. Mapa de susceptibilidade a deslizamentos na região metropolitana do Recife. Recife, 2007. 151 p. Tese de Doutorado (Doutorado em Geociências) - Centro de Tecnologia e Geociências, Universidade Federal de Pernambuco.

PINTO, R.C.; PASSOS, E.; CANEPARO, S.C. Mapeamento de suscetibilidade aos movimentos de massa com uso da Avaliação Multicritério pelo método da Média Ponderada Ordenada. Caderno de Geografia, v.25, n. 43, p. 116-143, 2015.

PÔSSA, E.M. \& VENTORINI, S.E. Expansão urbana para áreas de risco de inundação e de movimento d emassa: o estudo no município de São João Del-Rei - MG. Caderno prudentino de Geografia, Presidente Prudente, n. 36, v. 2, 49-67 p., 2014.

PRINA, B.Z. \& TRENTIN, R. Metodologia para o mapeamento de áreas suscetíveis a inundação: estudo de caso para o município de Jaguari/RS. In: XXVI CONGRESSO BRASILEIRO DE CARTOGRAFIA, V CONGRESSO BRASILEIRO DE GEOPROCESSAMENTO E XXV EXPOSICARTA. 2014, Gramado. Resumo Expandido...Gramado: Sociedade Brasileira de Cartografia, p. 1-13. 2014.

SADECK, L.W.R.; SOUZA. A.A.A. DE.; SILVA, L.C.T. DA. Mapeamento de zonas de risco as inundações no município de Belém - PA. VI Encontro Nacional de Anppas. Belém PA. 2012.

SAITO, S. Estudo analítico da susceptibilidade a escorregamentos e quedas de blocos no maciço central de Florianópolis-SC. Florianópolis, 2004. 133 p. Dissertação (Mestrado em Geografia) - Universidade Federal de Santa Catarina - UFSC.

SILVA, J.P. Mapeamento de inundações no Brasil: proposta de gestão ambiental através de um sistema de informações geográficas. In: IX SEMINÁRIO DE PÓS-GRADUAÇÃO EM GEOGRAFIA DA UNESP RIO CLARO, 2009, Rio Claro. Resumos Expandidos...São Paulo: Universidade Estadual Paulista, 2009.

SILVA, P.M. DA.; MIRANDA, F.P. DE.; LANDAU, L. Mapeamento dos índices de sensibilidade fluvial a derrames de óleo mediante análise espaço-temporal do fenômeno de inundação em Coari (AM). In: SIMPÓSIO BRASILEIRO DE SENSORIAMENTO REMOTO XVII. Resumo Expandido...João Pessoa, PB INPE: Instituto Nacional de Pesquisas Espaciais, 2015, p. 0407-0414.
SILVA JÚNIOR, O.M. \& SZLAFSZTEIN, C.F. Análise de risco como critério a gestão do território: um estudo do plano diretor do município de Alenquer (PA). Olam: Ciência \& Tecnologia, v. 10, p. 1-29, 2010a.

SILVA JÚNIOR, O.M. \& SZLAFSZTEIN, C.F. Metodologia de análise de risco a inundação como critério a gestão do território em pequenas cidades amazônicas: o caso de Alenquer - PA. In: XV Simpósio da Sociedade de Especialistas Latino-americano em Sensoriamento Remoto. Caiena. Anais of Symposium Selper. 2012.

SIMÕES, P.M.L. \& OLIVEIRA, C.V. Avaliação da susceptibilidade à erosão e aos movimentos de massa no município de Ibirité. Geonomos, v. 22, p. 22-30, 2014.

TAUBENBOCK, H.; POST, J.; ROTH, A.; ZOSSEDER, K.; STRUNZ, G.; DECH, S. A conceptual vulnerability and risk framework as outline to identify capabilities of remote sensing. Natural Hazards and Earth System Sciences, v. 8, p. 409-420. 2008.

TOMINAGA, L.K. Avaliação de metologia de risco a escorregamento: aplicação de um ensaio em Ubatuba, SP. São Paulo, 2007. 240 p. Tese (Doutorado em Geografia Física) - Departamento de Geografia, Faculdade de Filosofia, Letras e Ciências Humanas. USP.

UNDP - UNITED NATIONS DEVELOPMENT PROGRAM. A Global Report. Reducing disaster risk: a challenge for development. New York - USA. ISBN 92-1-126160-0. Disponível em: http://www.preventionweb.net/files /1096_rdrenglish.pdf. Acessado em: 20Ago2015. 2004.

VESTANA, L.R. Mapeamento da susceptibilidade a deslizamentos na bacia hidrográfica do Caeté, Alfredo Wagner/SC. Revista de Geografia da UFC (doi: 10.4215/RM2010.0919.0013). v. 9, n. 19, p. 175-190, 2010.

VIEIRA, I.M.; KARMIERCZAK, M.L.; MALTA, F.J.N.C. Proposta metodológica para identificação de áreas de risco de movimentos de massa em áreas de ocupação urbana. Estudo de caso: Campo do Jordão, SP. 2005. Goiânia. In: XII SIMPÓSIO BRASILEIRO DE SENSORIAMENTO REMOTO. Resumos Expandidos... Goiânia: Instituto Nacional de Pesquisas Espaciais, 2005. p. 3935-3942.

ZADEH, L.A. Fuzzy sets. Information and Control. California - USA. v. 8, p. 338-353. 1965.

Submetido em 12 de setembro de 2016 Aceito em 16 de novembro de 2016 